\title{
Immune Cell Populations in the Broiler Ileum Exhibit Differential Cytokine Profiles in Response to Lipid Source and Peroxidation
}

\section{DOI:10.31274/air.11909}

\author{
Krysten Fries-Craft, Graduate Research Assistant; \\ Brian J. Kerr, USDA-ARS-National Laboratory for \\ Agriculture and the Environment; \\ Elizabeth Bobeck, Assistant Professor; \\ Department of Animal Science, Iowa State University
}

\section{Summary and Implications}

Used restaurant oil offers a sustainable and affordable energy source in broiler diets but variable lipid composition and the presence of harmful peroxidation products may alter intestinal immunity. The objective of this study was to evaluate the effects of feeding different lipid sources with variable peroxidation statuses on immune cell populations producing interleukin-6 (IL6) and interferon- $\gamma$ (IFNG) in the broiler ileum. Two hundred broilers were fed diets with 5\% inclusion of control or peroxidized palm, soybean, flaxseed or fish oil in a $4 \times 2$ factorial treatment design. At $21 \mathrm{~d}, 2$ birds/ treatment were euthanized for ileum collection and immune cell populations were analyzed by RNAscope- in situ hybridization (ISH). Ileal production of IL6 increased $85.8 \%$ by feeding peroxidized flaxseed oil while $I F N G-$ producing cells were increased $55.1-59.9 \%$ by feeding either control or peroxidized soybean oil $(P \leq 0.05)$. Feeding peroxidized lipid generally reduced $C D 3^{+} \mathrm{T}$ cells not producing either IL6 or IFNG by $14.9-39.0 \%(P \leq 0.05)$. Overall, these results suggest that IL6 and IFNG have differential responses to lipid source and peroxidation while lipid peroxidation negatively impacts $\mathrm{T}$ cell presence in the broiler chicken ileum. Inflammatory outcomes observed in broilers fed peroxidized flaxseed oil suggest that yellow grease containing this type of oil may detrimentally impact broilers, while soybean oil generally contributes to intestinal inflammation regardless of heat exposure.

\section{Introduction}

Implementation of restaurant oil, i.e. yellow grease, in poultry diets can increase energy content while reducing production costs and promoting sustainability in the food chain. Yellow grease, a mixture of oils, has been repeatedly exposed to high temperatures that may contribute to the formation of peroxidation products like aldehydes and hydroperoxides. Polyunsaturated fatty acids (PUFA), such as those present in soybean, flaxseed, and fish oil, are susceptible to the formation of harmful and potentially inflammatory peroxidation products, while saturated fats like those found in palm oil are less susceptible. In addition to health considerations associated with repeated and prolonged heat exposure, the lipid composition of yellow grease may alter broiler immunity as lipid sources high in omega-6 PUFA (e.g. soybean oil) are associated with proinflammatory states while those with a higher prevalence of omega-3 PUFA (e.g. flaxseed and fish oil) are considered to be anti-inflammatory. In this study, RNAscope-ISH was used to examine tissue-level immune responses to control vs. peroxidized palm, soybean, flaxseed, and fish oil in the broiler ileum by showing the simultaneous RNA expression of a $\mathrm{T}$ cell marker $(C D 3)$ with inflammatory cytokines IL6 or IFNG.

\section{Materials and Methods}

All animal procedures were approved by the Institutional Animal Care and Use Committee at Iowa State University. A total of 200 1d-old broilers were housed in battery cages ( 5 birds/ cage) and assigned to 1 of 4 diets in a $4 \times 2$ factorial arrangement of lipid source (palm, soybean, flaxseed, or fish oil) and peroxidation status (control or peroxidized) after a $3 \mathrm{~d}$ acclimation period. Lipids were included in the diet at 5\% and peroxidation was achieved by thermally processing the lipids at $90^{\circ} \mathrm{C}$ with continuous air infusion (3L/min) for $72 \mathrm{~h}$. At $\mathrm{d} 21,2 \mathrm{birds} /$ treatment were euthanized for tissue collection.

To visualize cytokine and T cell RNA expression within intestinal tissue, ileum sections were fixed in $10 \%$ neutral buffered formalin for $24 \mathrm{~h}$ prior to RNAscope analysis using the ACDbio RNAscope 2.5 HD Assay-Red kit (Advanced Cell Diagnostics, Newark, CA) in accordance with the manufacturer's instructions. Custom speciesspecific RNAscope probes based on Genbank sequences were used to visualize CD3, IL6, and IFNG RNA in the broiler ileum. Both cytokine RNA signals were measured alongside $C D 3 \mathrm{~T}$ cell probes to identify potential cell types contributing to each inflammatory marker. HALO software (Indica Labs, Corrales, NM) was used to analyze RNAscope outputs by approximating the location of individual cells and describing the percentage of cells expressing each RNAscope probe signal for 6 fields/ birds (12 fields/ treatment). Outputs from the software include populations expressing only the cytokine or $\mathrm{T}$ cell marker in addition to those expressing both markers simultaneously or not at all.

Cell population data provided by the HALO software was analyzed in SAS 9.4 using the MIXED procedure as

Works produced by employees of the U.S. Government as part of their official duties are not copyrighted within the U.S. The content of this document is not copyrighted. 
repeated measures with a compound symmetry covariance structure and individual bird as the subject. Fixed effects were lipid source, peroxidation status, and the lipid $\times$ peroxidation interaction with significance denoted at $P \leq$ 0.05 .

\section{Results and Discussion}

Feeding peroxidized flaxseed oil increased the number of cells producing IL6 in the broiler ileum by $85.8 \%$ while reducing overall $C D 3^{+} \mathrm{T}$ cells by $39.0 \%$ compared to control flaxseed oil ( $P=0.03$ and 0.02 ; Figure 1A,B). Despite the reduction in T cells, the percentage of these cells producing IL6 increased by $79.0 \%$ in the ileum of broilers fed peroxidized vs. control flaxseed oil $(P=0.06$; Figure $1 C)$. In general, feeding peroxidized lipid increased the percentage of cells that could not be identified by $C D 3$ or IL6 expression in the ileum by $18.4 \%(P=0.02)$ but this change was not specific to peroxidized flaxseed oil (Figure 1D).

For $I F N G$, the main effect of lipid source impacted cytokine expression more than lipid source and peroxidation status combined. Broilers fed soybean oil-containing diets had 55.1-59.9\% more ileal cells producing IFNG compared to those fed palm and fish oils, regardless of peroxidation status $(P=0.007$; Figure $2 \mathrm{~A})$. While overall T cells were decreased $14.9 \%$ by the main effect of peroxidation $(P=$ 0.03 ), populations of T cells producing $I F N G$ were increased by 58.9-67.1\% in soybean oil-fed birds compared to their counterparts on palm and fish oil diets $(P=0.02$; Figure 2B,C). Like outcomes observed in IL6-producing cells, the main effect of peroxidation increased ileal cells that could not be identified by $C D 3$ or $I F N G$ expression by $24.0 \%(P=0.01$; Figure $2 \mathrm{D})$.

Based on these outcomes, sources of yellow grease with a high percentage of flaxseed oil may result in increased production of inflammatory IL6 in the broiler ileum that can be mitigated if control (unheated) oil is fed. While the lipid source and peroxidation status impacted IL6 production, soybean oil increased IFNG regardless of peroxidation. These results suggest that soybean oil may contribute to a generalized pro-inflammatory state in the broiler ileum.

The reduction in $\mathrm{T}$ cells observed when measured along with both $I L 6$ and IFNG in broilers fed peroxidized lipids suggests that compounds produced during peroxidation alter local $\mathrm{T}$ cell populations or reduce ileal lymphocyte recruitment. Further research is needed to specify which compounds may be responsible for this outcome and how $\mathrm{T}$ cell populations altered by feeding peroxidized lipids may contribute to broiler health and subsequent performance.

\section{Acknowledgements}

The authors would like to thank the staff at the Iowa State University Poultry Research and Teaching Farm for animal care support during this study. 
A.

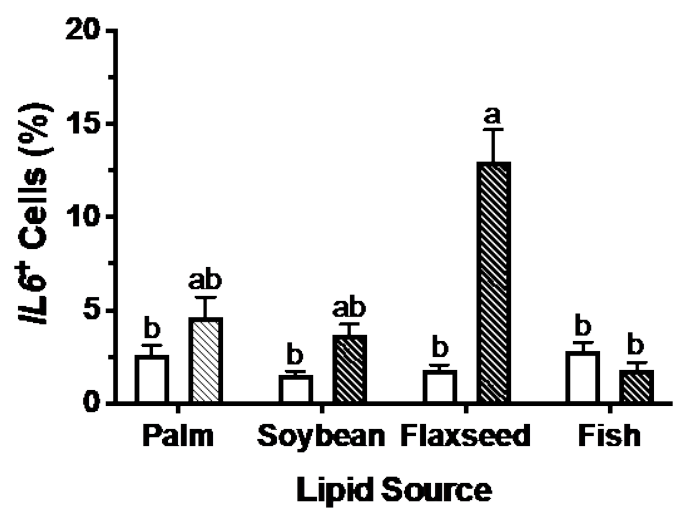

c.

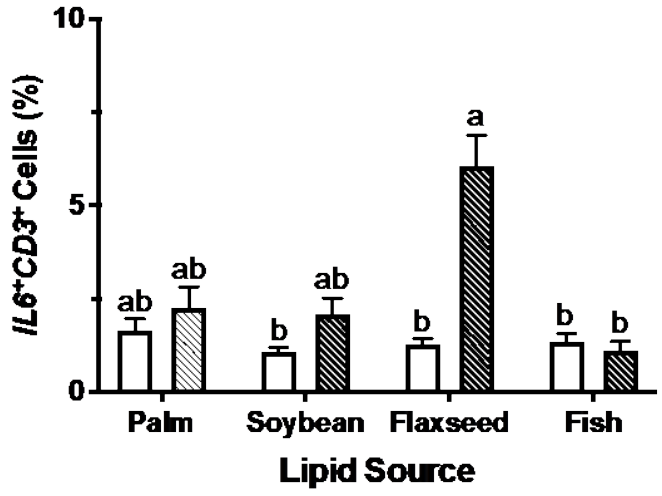

B.

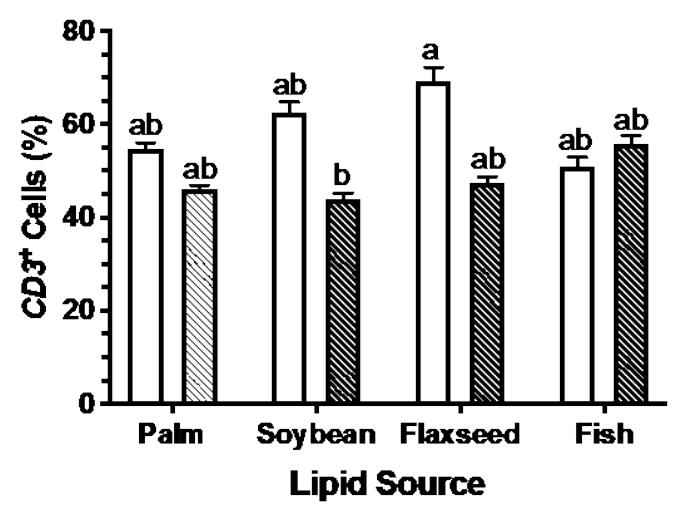

D.

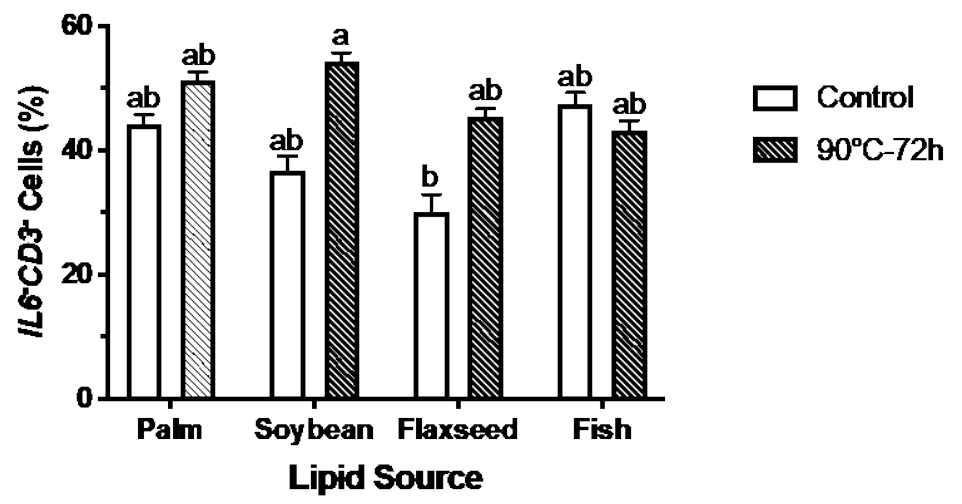

Figure 1: Populations of (A) cells producing $I L 6,(\mathbf{B}) C D 3^{+} \mathrm{T}$ cells, $(\mathbf{C}) \mathrm{T}$ cells producing $I L 6$, and (D) cells that could not be identified by either marker in the ileum of broilers fed control or peroxidized palm, soybean, flaxseed, or fish oils. Data represent the mean percentage of each cell population observed in 6 ileal fields in 2 birds/ treatment \pm SEM. Bars labeled with different letters are significantly different $(P \leq 0.05)$. 
A.

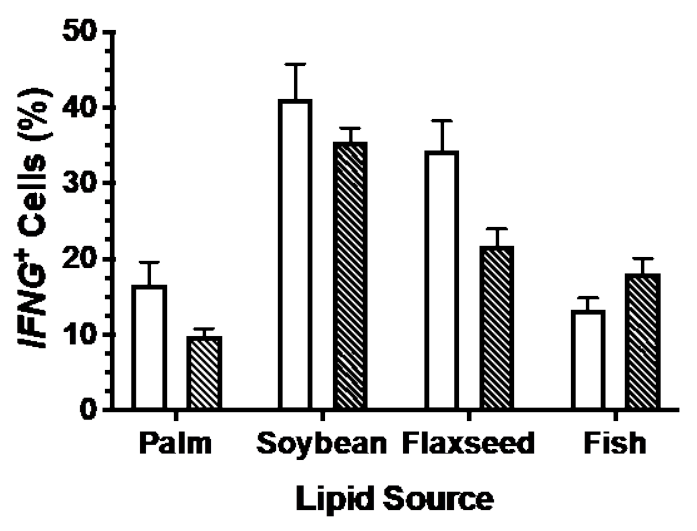

C.

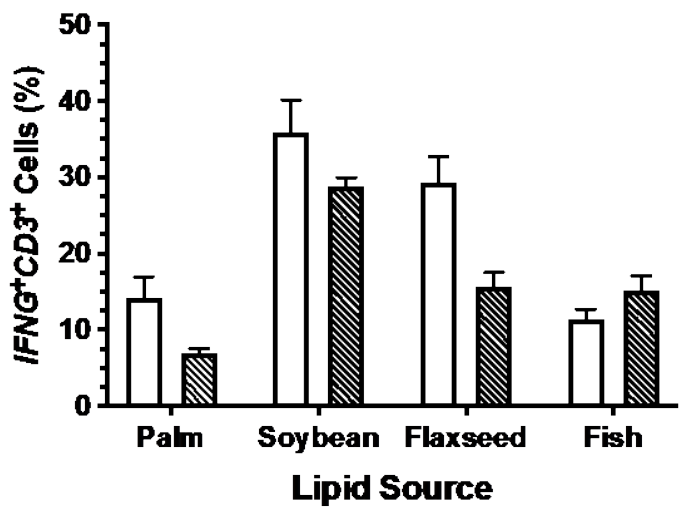

B.

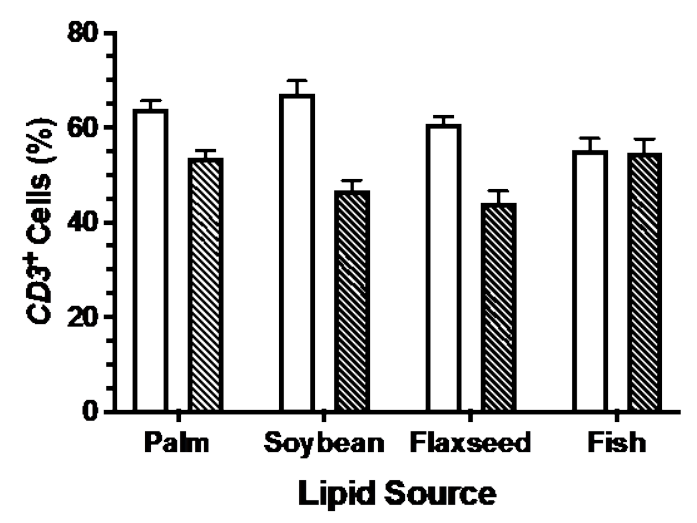

D.

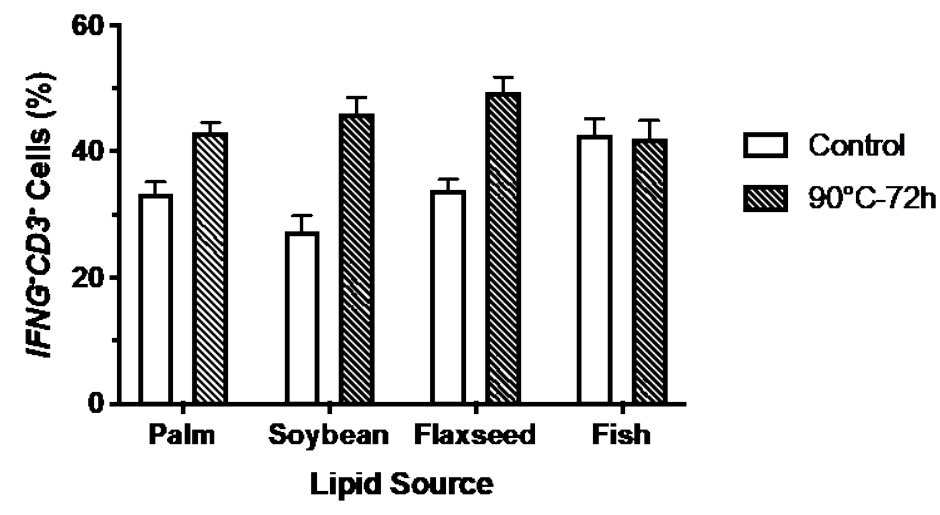

Figure 2: Populations of (A) cells producing $I F N G$, (B) $C D 3^{+} \mathrm{T}$ cells, $(\mathbf{C}) \mathrm{T}$ cells producing $I F N G$, and (D) cells that could not be identified by either marker in the ileum of broilers fed control or peroxidized palm, soybean, flaxseed, or fish oils. Data represent the mean percentage of each cell population observed in 6 ileal fields in 2 birds/ treatment \pm SEM. Bars labeled with different letters are significantly different $(P \leq 0.05)$. 